INPLASY

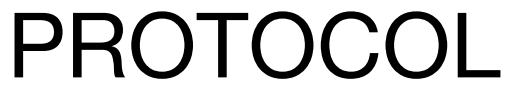

To cite: Yang et al. Inhalational versus Intravenous

Maintenance of Anesthesia for Emergence Delirium in Adults: A Meta-analysis and Trial Sequential Analysis. Inplasy protocol 202070089. doi: 10.37766/inplasy2020.7.0089

Received: 20 July 2020

Published: 20 July 2020

Corresponding author: Bing Chen

chenbing2050@163.com

Author Affiliation:

The Second Affiliated Hospital of Chongqing Medical

University

Support: None

Review Stage at time of this submission: Preliminary searches.

Conflicts of interest:

None.

\section{Inhalational versus Intravenous Maintenance of Anesthesia for Emergence Delirium in Adults: A Meta-analysis and Trial Sequential Analysis}

Yang, Y1; Feng, L2; Ji, CC³; Lu, K4; Chen, Y5; Chen, B6.

Review question / Objective: To compare the incidence of emergence delirium in adults between inhalational and intravenous maintenance. (1) Patients: adult surgical patients intend to extubation and emergence at operation room, postanesthesia care unit or intensive care unit after intravenous or inhalational anesthesia maintenance. (2) Intervention: inhalational maintenance of anesthesia. (3) Comparator: propofol-based maintenance of anesthesia. (4) Outcomes: the incidence of emergence delirium occurred at the period from unconsciousness to full wakefulness after operation. (5) Study design: RCT, cohort study, case-control study.

INPLASY registration number: This protocol was registered with the International Platform of Registered Systematic Review and Meta-Analysis Protocols (INPLASY) on 20 July 2020 and was last updated on 20 July 2020 (registration number INPLASY202070089).

\section{INTRODUCTION}

Review question / Objective: To compare the incidence of emergence delirium in adults between inhalational and intravenous maintenance. (1) Patients: adult surgical patients intend to extubation and emergence at operation room, postanesthesia care unit or intensive care unit after intravenous or inhalational anesthesia maintenance. (2) Intervention: inhalational maintenance of anesthesia. (3) Comparator: propofol-based maintenance of anesthesia. (4) Outcomes: the incidence of emergence delirium occurred at the period from unconsciousness to full wakefulness after operation. (5) Study 
design: RCT, cohort study, case-control study.

Condition being studied: Emergence delirium (ED) is characterized by agitation, restlessness and hyperactivity, therefore, it also names emergence agitation or emergence excitement. ED increases the risk for injury, self-extubation, hemorrhages and prolonged hospitalization. Inhalational agents and propofol-based intravenous anesthesia are two commonly used maintenance of general anesthesia, which one is safer has been investigated by anesthesiologists for many years. Many studies and the European Society of Anesthesiology evidence-based and consensus-based guideline on postoperative delirium have recognized inhalational maintenance of anesthesia as a risk factor for pediatric ED. However, ED in adults between inhalational and intravenous maintenance of anesthesia is undefined.

\section{METHODS}

Search strategy: Pubmed and Cochrane Library \#1 MeSH descriptor: [Anesthesia, Intravenous] explode all trees \#2 MeSH descriptor: [Anesthesia, Inhalation] explode all trees \#3 MeSH descriptor: [Anesthetics, Inhalation] explode all trees \#4 MeSH descriptor: [Anesthetics, Intravenous] explode all trees \#5 an?esthe* [TIAB] \#6 intravenous [TIAB] OR inhalation* [TIAB] OR volatile [TIAB] \#7 \#5 AND \#6\#8 TIVA [TIAB] OR propofol [TIAB] OR sevoflurane [TIAB] OR enflurane [TIAB] OR isoflurane [TIAB] OR desflurane [TIAB] OR halothane [TIAB] \#9 \#1 OR \#2 OR \#3 OR \#4 OR \#7 OR \#8 \#10 delirium [TIAB] OR agitation [TIAB] OR excitement [TIAB] OR inadequate [TIAB] \#11 emergence [TIAB] \#12 \#9 AND \#10 AND \#11 Medline \#1 TS=(Anesthetics, Inhalation) OR TS=(Anesthetics, Intravenous) OR TS=(Anesthesia, Intravenous) OR TS=(Anesthesia, Inhalation) \#2 TS=(an?esthe $\left.{ }^{*}\right)$ \#3 TS=(intravenous) OR TS=(inhalation*) OR TS=(volatile) \#4 \#2 AND \#3 \#5 TS=(TIVA) OR TS=(propofol) OR TS=(sevoflurane) OR TS=(enflurane) OR TS=(isoflurane) OR TS=(desflurane) OR TS=(halothane) \#6 \#1
OR \#4 OR \#5 \#7 TS=(delirium) OR TS=(agitation) OR TS=(excitement) OR TS=(inadequate) \#8 TS=(emergence) \#9 \#6 AND \#7 AND \#8 Embase \#1 intravenous anesthesia [emtree term-exploded] OR inhalation anesthesia [emtree termexploded] \#2 an?esthe* [ti,ab,kw] \#3 intravenous [ti,ab,kw] OR inhalation* [ti,ab,kw] OR volatile [ti,ab,kw] \#4 \#2 AND \#3 \#5 TIVA [ti,ab,kw] OR propofol [ti,ab,kw] OR sevoflurane [ti,ab,kw] OR enflurane [ti,ab,kw] OR isoflurane [ti,ab,kw] OR desflurane [ti,ab,kw] OR halothane [ti,ab,kw] \#6 \#1 OR \#4 OR \#5 \#7 delirium [ti,ab,kw] OR agitation [ti,ab,kw] OR excitement [ti,ab,kw] OR inadequate [ti,ab,kw] \#8 emergence [ti,ab,kw] \#9 \#6 AND \#7 AND \#8.

Participant or population: Adult surgical patients intend to extubation and emergence at operation room, postanesthesia care unit or intensive care unit after intravenous or inhalational anesthesia maintenance.

Intervention: Inhalational maintenance of anesthesia.

Comparator: Propofol-based maintenance of anesthesia.

Study designs to be included: RCT, cohort study, case-control study.

Eligibility criteria: Studies met the PICOS (patients, intervention, comparator, outcome, study design) criteria. (1) Patients: adult surgical patients intend to extubation and emergence at operation room, postanesthesia care unit or intensive care unit (ICU) after intravenous or inhalational anesthesia maintenance. (2) Intervention: inhalational maintenance of anesthesia. (3) Comparator: propofol-based maintenance of anesthesia. (4) Outcomes: the incidence of ED occurred at the period from unconsciousness to full wakefulness after operation. (5) Study design: RCT, cohort study, case-control study.

Information sources: Embase, PubMed, Medline and the Cochrane Library from inception to July 2020. 
Main outcome(s): The incidence of emergence delirium occurred at the period from unconsciousness to full wakefulness after operation. Because there are many scales to diagnosis emergence delirium, so the incidence of emergence delirium will be directly extracted from the included studies.

\section{Additional outcome(s): None.}

Quality assessment / Risk of bias analysis: The quality of RCTs will be assessed according to the guidelines in the Cochrane Handbook for Systematic Reviews of Interventions to create a "risk of bias" table that included the following contents: details on methods of random sequence generation, allocation concealment, blinding, incomplete outcome data, selective outcome reporting, and other bias. The overall quality of each study will be evaluated as "low risk of bias", "high risk of bias", or "unclear risk of bias". Moreover, the RCTs will be quantitatively assessed by a modified Jadad 7-point scale and the Jadad score $\geq 4$ is considered to be of high-quality. The quality of observational studies will be evaluated using the Newcastle-Ottawa Scale (NOS) with a maximum total score of 9 that will be judged at population selection, comparability and outcomes. A NOS score $\geq 7$ is regarded as high-quality. If the number of included studies $\geq 10$, the publication bias will be assessed by funnel plot.

Strategy of data synthesis: The metaanalysis will be conducted by Review Manager software 5.4. Risk ratio (RR) with $95 \%$ confidence intervals (Cls) will be used to assess the incidence of emergence delirium. Statistical heterogeneity will be assessed by $P$ and 12 . When $12<50 \%$ and $P$ $>0.1$, a fixed-effects model will be applied; otherwise, a random-effects model will be applied. The Mantel-Haenszel methods will be used to combine separate statistics. $P$ values less than 0.05 will be considered statistically significant. Trial sequential analysis (TSA) software 0.9 .5 .10 will be used to examine the reliability and conclusiveness of the available evidence according to the previous meta-analysis. Two-sided tests with a type I error of $5 \%$, a power of $80 \%$, a low bias based relative risk reduction and an incidence of ED of $5 \%$ in control will be used to calculate the RIS.

Subgroup analysis: Subgroup analysis will be conducted based on the existed features in studies: (1) RCTs vs. Non-RCTs; (2) different screening scales for ED; (3) different inhalational anesthetics (sevoflurane, enflurane, isoflurane, desflurane, halothane); (4) studies were of high-quality.

Sensibility analysis: Sensitivity analysis will be conducted by omitting one study in turn.

Language: English.

Country(ies) involved: China.

Keywords: Emergence delirium; intravenous anesthesia; inhalational anesthesia; adults; meta-analysis.

Contributions of each author:

Author 1 - Yong Yang - The author collected and analyzed the data, and wrote the manuscript.

Author 2 - Lin Feng - The author collected and analyzed the data, and wrote the manuscript.

Author 3 - Chengcheng Ji - The author collected and analyzed the data.

Author 4 - Kaizhi Lu - The author revised data and manuscript.

Author 5 - Yang Chen - The author revised the data and proofread the manuscript.

Author 6 - Bing Chen - The author designed the study, wrote and proofread the manuscript. 